\title{
ANÁLISIS COMPARATIVO DE LAS ASIGNATURAS TIC EN LA FORMACIÓN INICIAL DE PROFESORES EN CHILE ENTRE 2012 Y 2018
}

\author{
COMPARATIVE ANALYSIS OF ICT SUBJECTS IN INITIAL TEACHER TRAINING IN \\ CHILE BETWEEN 2012 AND 2018
}

\author{
Hugo Gonzalo Tapia Silva (*) \\ Universidad de La Serena \\ Chile \\ Karla Alejandra Campaña Vilo \\ Universidad de La Serena \\ Chile \\ Rodrigo Fernando Castillo Robledo \\ Departamento de Educación \\ Universidad de La Serena \\ Chile
}

Instituto de Investigación Multidisciplinar de Ciencias y Tecnología

Unidad de Gestión Integrada de las Pedagogías

\section{Resumen}

La presencia de las TIC en la formación inicial docente debería responder a diferentes factores, como las políticas públicas y las perspectivas teóricas. En cuanto crece la importancia de las TIC en educación, se esperaría que los programas de formación aumentaran la presencia de las tecnologías. La presente investigación busca identificar si hubo un cambio en el número de asignaturas TIC en carreras de pedagogía entre los años 2012 ( $n=212$ ) y 2018 ( $n=237)$ en Chile, a través de la comparación de las mallas curriculares de los programas de formación. Se concluye que existe una variación porcentual positiva en el número de carreras con al menos 1 asignatura TIC (12,4\%), en el promedio de asignaturas TIC (29,5\%), en el número de programas que incluyen 2 asignaturas TIC, y diferencia en el número de programas con presencia de las TIC entre grupos disciplinares.

Palabras clave: Asignaturas; TIC; formación; profesores; Chile.

\section{Abstract}

Hugo Gonzalo Tapia Silva

Universidad de la Serena, Chile

Instituto de Investigación Multidisciplinar de Ciencias y Tecnología

Benavente 980 - La Serena, Chile

Correo de contacto: hgtapia@gmail.com

(C)2010, Perspectiva Educacional Http://www.perspectivaeducacional.cl

RECIBIDO: 56.04 .2019 ACEPTADO: 25.09 .2019

DOI: 10.4151/07189729-Vol.59-Iss.1-Art.963
The presence of ICT in initial teacher education should respond to different factors, such as public policies and theoretical perspectives. While the importance of ICT in education grows, it would be expected that training programs increase the presence of technologies. The purpose of this article is to identify if there was a change in Chile in the amount of ICT subjects in initial teacher training between the years 2012 ( $n=$ 212) and 2018 ( $n=237$ ), through the comparison of their curricula. It is concluded that there is a positive percentage variation in the number of programs with at least 1 ICT course (12.4\%), in the average of ICT courses $(29.5 \%)$, in the number of programs that include 2 ICT courses, and a difference in the number of courses with presence of ICT among disciplinary groups.

Keywords: Courses; ICT; Training; Teachers; Chile. 


\section{Introducción}

La incorporación de las Tecnologías de la Información y las Comunicaciones (en adelante, TIC) en los procesos de aprendizaje, es un tema complejo que involucra acciones a nivel político, universitario, escolar y de los privados. Todos estos esfuerzos se enfrentan a diversos problemas, siendo uno de los más importantes la necesidad de contar con un profesorado con competencias digitales que les permitan la integración didáctica de las TIC en sus clases.

Las investigaciones evidencian las TIC como una inquietud en el desarrollo de la informática educativa y en el aprendizaje, tanto en el mundo académico como escolar (Jara, 2007; Jara \& Hinostroza, 2014). Sin embargo, esta preocupación no se relaciona con la presencia de las TIC en el itinerario formativo de cada institución de educación superior que ofrece la carrera de pedagogía.

Actualmente, las organizaciones han iniciado procesos de rediseño curricular de las carreras de pedagogía, con nuevos estándares formativos y sistemas de aseguramiento de la calidad, como, por ejemplo, la Evaluación Nacional Diagnóstica. En este contexto, resulta importante identificar si existe una mayor presencia de las TIC en los planes de formación conforme a las exigencias del sistema y a las demandas de la sociedad del siglo XXI.

La presente investigación busca identificar si la integración de las TIC en los planes de formación de carreras de pedagogía en Chile ha tenido alguna variación en número y promedio de asignaturas TIC, tanto a nivel general como por disciplina formativa en los últimos años. Para ello, realizamos una comparación de la presencia de asignaturas que abordan expresamente las TIC en las mallas curriculares entre los años 2012 y 2018, para verificar si ha aumentado la presencia de las TIC en la Formación Inicial de Profesores (en adelante, FIP) en un período en que algunas carreras han renovado su currículo y se han sometido a procesos de acreditación.

Los resultados de esta investigación suponen un dato importante al evaluar la integración de las TIC en los programas formativos de profesores. Permiten identificar, en primer lugar, si ha variado el número de asignaturas TIC en los programas de formación para adecuarse a los requerimientos actuales. En segundo lugar, ayudarán a reconocer cuánta es, si es que existe, la diferencia en el número de asignaturas TIC entre las carreras de pedagogía de disciplinas formativas distintas. 


\subsection{La integración de las TIC en la FIP}

La integración de las TIC en los programas de FIP es diversa y depende de varios factores. En primer lugar, y siguiendo a Rizza (2011), podemos encontrar cuatro niveles distintos de integración de las TIC vinculados a las políticas públicas de un país:

- Nivel 1: Las políticas públicas realizan recomendaciones, pero no se requiere formación.

- Nivel 2: Las políticas públicas proponen estándares obligatorios de capacitación y acreditación para los programas de formación.

- Nivel 3: Las políticas públicas implementan un marco de competencias.

- Nivel 4: Las políticas públicas establecen certificación para el profesorado.

En segundo lugar, la manera de integrar las TIC en los programas de formación también depende del enfoque formativo que se utilice. Podemos reconocer tres enfoques de la formación en TIC: a) formación con uno o más cursos específicos; b) formación de carácter transversal, sin una asignatura específica; y c) formación mixta, que considera los dos primeros enfoques. La elección de cada una de ellas depende, entre otros aspectos, de las orientaciones del currículo, el tiempo establecido o los recursos disponibles.

En tercer lugar, dependería del modelo formativo del programa: consecutivo o simultáneo (Ávalos, 2010; Brun \& Hinostroza, 2011; Marcelo, 2016; Rebolledo Gómez, 2015). El modelo consecutivo consta de una primera etapa en que se estudia una licenciatura (Matemáticas, Artes u otras), y una segunda con la formación específica para la profesión docente. Mientras que, en el modelo simultáneo, un programa de formación aborda tanto los contenidos de la disciplina como los pedagógicos, con una extensión que varía según las políticas educativas o de los centros de formación. En el caso chileno, el modelo formativo habitual es el simultáneo, en el que se debería abordar la formación en TIC.

A nivel internacional existen experiencias y modelos formativos que presentan variadas respuestas a la preocupación de integrar las TIC en los planes de estudio para la formación de profesores. En el caso de España, se observa una presencia dispar de las asignaturas TIC, con carreras que no incluyen asignaturas y carreras que sí lo hacen (Herrada Valverde \& Herrada Valverde, 2011). Dentro de estas últimas, se encuentran carreras que incluyen habilidades TIC de manera transversal, las que se deben adquirir para obtener el título universitario (García Valcárcel \& Martín del Pozo, 2016). En el caso de Noruega, hay programas de formación de profesores que no cuentan con cursos específicos sobre el uso de las TIC, debiendo incorporar estos contenidos en asignaturas como las de Didáctica (Instefjord \& Munthe, 2016; Røkenes \& 
Krumsvik, 2016). La formación de profesores en Finlandia, por su parte, incluye un curso al inicio de la formación Ilamado Pedagogical views of ICT in Education (Kontkanen, 2018).

En el ámbito latinoamericano, destacamos el caso de Uruguay y su política de integración de las TIC en educación. Esta política ha estado presente desde el año 1985 con diversas iniciativas. No obstante, la formación de profesores en aspectos ligados a la integración didáctica de las TIC presenta carencias importantes (Martínez, Leite \& Reis, 2016).

Enochsson y Rizza (2009) identificaron en un informe de la Organización para la Cooperación y el Desarrollo Económicos (OCDE) que, de un total de 120 instituciones en Estados Unidos de América, el 62\% ofrecía cursos específicos sobre uso de las TIC para profesores. En un informe posterior, Rizza (2011) señala que varios países disponen estándares TIC, requisitos de acreditación y de certificación del profesorado. Este contexto ha ido definiendo, como señalan Tondeur, Van Braak, Siddiq y Scherer (2016), que muchos centros de formación comenzaran a incorporar han incorporado cursos sobre TIC, de preferencia introductorios al uso de ellas, con énfasis en el desarrollo de conocimiento y habilidades. Esta estrategia ha sido permanente en el plano internacional desde los años 1990 (Mouza, Karchmer-Klein, Nandakumar, Yilmaz Ozden \& $\mathrm{Hu}, 2014)$.

Respecto a lo anterior, Flores-Lueg y Roig Vila (2016) aseguran que una sola asignatura TIC en un programa de FIP no es suficiente para obtener un nivel de conocimiento para la integración de las TIC en las prácticas pedagógicas. Coinciden Adnan y Tondeur (2018) en esta apreciación. En esta misma línea, Forkosh-Baruch (2018) identifica que la presencia de cursos TIC en la formación de futuros profesores permitiría, junto con su aplicación en las prácticas en contextos laborales, contar con profesionales con una mirada integral de la presencia de las tecnologías en el aula. Mouza et al. (2014) complementan lo anterior, señalando que los cursos TIC, junto a cursos de Didáctica, favorecerán sus futuras prácticas pedagógicas con integración de tecnología. Lamentablemente, en varios países, incluido Chile, la incorporación ha resultado desigual (Silva, Gros, Garrido \& Rodríguez, 2006).

\subsection{Las TIC en la FIP en Chile}

De acuerdo a los datos recientes de la Comisión Nacional de Acreditación (en adelante, CNA) se ofertan más de 500 programas del área de educación, la mayoría bajo un modelo simultáneo, en universidades estatales, privadas e institutos profesionales que otorgan licenciatura y/o título profesional. Un 64\% (algo más de 320) de estas carreras se encuentran acreditadas, un 19\% no acreditadas y un 17\% nunca se ha sometido a procesos de acreditación (CNA, 2018). 
La presencia de las TIC en el contexto de un modelo simultáneo ha considerado, entre otros factores, las orientaciones de las políticas públicas a través de la iniciativa ENLACES (actualmente, Centro de Innovación), los fundamentos pedagógicos de la presencia de las TIC o las especificidades del perfil de egreso o el modelo educativo de la institución formadora. Resulta especialmente importante este último factor, pues son estas instituciones quienes deciden, bajo criterios que aseguren aprendizajes comunes, la organización curricular de los programas de formación y su duración (Ávalos, 2010; Brun \& Hinostroza, 2011).

Para alcanzar resultados satisfactorios en la integración de las TIC en la FIP, 18 universidades chilenas pertenecientes a la Red de Asistencia Técnica de ENLACES desarrollaron en conjunto 6 proyectos, abordando, entre otros, el fortalecimiento del perfil de egreso de carreras de pedagogía y las didácticas específicas (Garrido, Gros, Rodríguez, Silva \& Nervi, 2008). A partir de ese trabajo, se identificaron algunos desafíos que permitirían profundizar en la ejecución de los proyectos, destacando la necesidad de actualizar los planes formativos y de vincular las prácticas de los formadores con los estándares TIC nacionales.

Por su parte, Brun e Hinostroza (2011) observaron las características del uso que se hace de las TIC en la formación de los profesores tras encuestar a 46 instituciones, 495 formadores, 164 profesores de práctica, 1.675 estudiantes, 233 graduados recientes y 50 expertos o técnicos TIC. Los resultados dan cuenta de las políticas institucionales, la infraestructura y recursos TIC, el soporte técnico, las creencias y competencias sobre el uso de las TIC y las prácticas de enseñanza y aprendizaje. En específico, al referirnos a las políticas institucionales y las actividades, se reconocen las siguientes características:

Tabla 1

Características de las políticas institucionales y de actividades de enseñanza y aprendizaje

Políticas institucionales Actividades de enseñanza y aprendizaje

- El uso de las TIC en la FIP no es • Uso básico, poco diverso y con un limitado transversal. set de herramientas digitales

- Competencias pedagógicas TIC no muy explícitas.

(principalmente proyectores y ordenadores).

- La formación en TIC de los formadores - Preponderancia de enseñanza y es mayoritariamente opcional. actividades de aprendizaje tradicionales.

- Solo un $54 \%$ de los formadores se ha - La frecuencia de actividades sobre cómo formado en TIC en los últimos 5 años. enseñar con TIC es menor que la de

- Baja presencia de estándares en las actividades en que solo el profesor las usa. evaluaciones.

- El uso de estándares no es una práctica habitual.

Fuente: Elaboración propia, a partir de Brun e Hinostroza (2011). 
En línea con lo anterior, Silva et al. (2006) concluyeron que los usos de las herramientas informáticas en los programas de formación se encuentran vinculados al conocimiento de su utilidad en el ámbito educativo, sea para planificar actividades con uso de TIC o en el uso de la tecnología para desarrollar recursos didácticos.

\subsection{Normas y propuestas para la integración de las TIC en la FIP en Chile}

Desde el año 1993, el programa ENLACES lideró el trabajo en el uso de las TIC en el ámbito escolar chileno (Muñoz \& Ortega, 2015). En un comienzo, se preocupó de garantizar la cobertura de equipos y recursos, para luego centrarse en alfabetizar digitalmente a los profesores en ejercicio. Posteriormente, en 2011 y 2013, lideró la medición de las habilidades TIC de los escolares.

Desde el punto de vista curricular, los esfuerzos se centraron en desarrollar una propuesta de incorporación de Estándares TIC en la FIP, basada en una exhaustiva revisión de experiencias internacionales (Ascencio Ojeda, Garay Aguilar \& Seguic Zeran, 2016; ENLACES, 2008), configurando una hoja de ruta para los programas de formación que deseen incorporar las TIC en el itinerario formativo, de manera transversal o como asignatura. Sin embargo, tal como establece Silva (2017), esta incorporación no ha permeado en los planes de formación docente de las instituciones de educación superior.

Los estándares orientadores genéricos y disciplinarios para la FIP desarrollados el año 2012 (Ministerio de Educación [MINEDUC], 2012a, 2012b, 2012c) y aún vigentes para las renovaciones curriculares de las carreras de pedagogía, incluyen el uso de las TIC como una de las habilidades básicas que se esperaría de un profesional egresado en el siglo XXI. En el caso de los criterios de acreditación para las carreras de pedagogía (CNA, 2007), se establece la existencia de un área de formación sobre el conocimiento de aspectos instrumentales para la docencia que debería incorporar el uso de TIC.

Una propuesta más reciente es la presentada por el Observatorio TIC en la Formación Inicial de Docentes, conformada por 13 universidades con programas de formación de profesores, que buscan ser el referente nacional sobre recursos y experiencias para la inclusión de las TIC en sus procesos formativos. En la misma línea, los nuevos criterios específicos de evaluación para la acreditación de programas regulares de pedagogía propuestos por la CNA (2017) dan cuenta de la exigencia en el uso de tecnología para los formadores de formadores y en las líneas de investigación relacionadas que sustentan el claustro académico de estas carreras. 
Otro elemento que considerar es la Agenda Digital 2020 (Gobierno de Chile, 2015). En ella se proponen 60 medidas enfocadas en el desarrollo digital de Chile en 5 ejes: Derechos para el desarrollo digital, Conectividad digital, Gobierno digital, Economía digital y Competencias digitales. En especial, este último eje posee como línea de acción mejorar la calidad de la educación mediante las tecnologías. En concreto, se propone "desarrollar un plan de acompañamiento a la innovación de la formación inicial docente con apoyo de las TIC" (Gobierno de Chile, 2015, p. 60).

No obstante este importante número de iniciativas, y en línea con lo expresado por Rizza (2011), las políticas públicas se ubican en el nivel 1 de integración de las TIC en la FIP. Esto supone un contexto en el que la formación en TIC de los futuros docentes puede ser muy diferente en cada carrera de pedagogía, incluso dentro de la misma disciplina, y, por tanto, su desempeño profesional puede ser diverso.

\subsection{Disciplina y formación en TIC}

La particularidad de cada disciplina podría implicar diferencias en el uso de las tecnologías, no solo en la frecuencia de uso, sino también en la perspectiva de su integración didáctica. Por ejemplo, como señalan Barton y Haydn (2006), los profesores de Historia utilizarían mayormente software genérico, es decir, que no ha sido diseñado para el aprendizaje, y visitas a sitios web específicos en sus clases. Los profesores de Ciencias usan software desarrollado especialmente para su asignatura (simulación, experimentación virtual, otros), es decir, materiales didácticos (Parcerisa, 2010).

En la misma línea, Webb y Cox (2004) presentan ejemplos de usos específicos más frecuentes de las TIC por parte de los profesores:

- simulación y modelado, en Ciencia y otras asignaturas;

- ambientes de modelado y otro tipo de software en Matemáticas;

- procesador de textos para Lenguaje y Literatura;

- uso de internet para aumentar la información de la disciplina;

- software de presentaciones para desarrollar exposiciones por parte de los estudiantes y habilidades de alfabetización;

- pizarras interactivas para promover la discusión en clase y habilidades para explicar y presentar información. 
Por tanto, el uso de las TIC difiere en el profesorado según su disciplina, pues cada uno de estos posee características y requerimientos que son propios (De Aldama \& Pozo, 2016; Howard, Chan \& Caputi, 2015; Labbé, Matamala, Astudillo \& Hinostroza, 2012). Considerar estas diferencias es, por tanto, fundamental al diseñar la adopción de las TIC en programas de formación de profesores.

Como señalan Arancibia Herrera, Casanova Seguel y Soto Caro (2016), la formación de profesores debe considerar de qué forma algunas concepciones inherentes a las disciplinas influyen en el modo de utilizar las TIC. La integración que se promueva de las TIC por parte de los formadores a través de las distintas actividades de aprendizaje fomentará el conocimiento y el desarrollo de cierto tipo de herramientas y actividades, con una didáctica asociada.

Los futuros profesores deben estar familiarizados con distintos usos que se pueden hacer de las TIC pues, en la medida de que empleen las tecnologías para la enseñanza de sus especialidades, estarán más preparados para adoptarlas y utilizarlas en sus clases (Gibson, Moline \& Dyck, 2011). En la misma línea, una investigación desarrollada por Chousa Cortés, Martínez-Figueira y RaposoRivas (2017), concluye que un mayor acercamiento a las TIC permite innovar en el uso de ellas, abordando cuestiones como, por ejemplo, apoyar a estudiantes con déficit atencional, mejorar acciones en atención, la lectoescritura, las matemáticas o habilidades sociales.

Finalmente, Garrido, Contreras y Miranda (2013) nos manifiestan que las casas formadoras de profesores debieran repensar su currículo formativo pues desarrollan una escasa innovación en el uso de las TIC.

\section{Metodología}

\subsection{Muestra}

La muestra de la investigación está compuesta por las mallas de carreras de pedagogía que cumplieran con los siguientes criterios: ser una carrera de pedagogía de educación media, que se imparta en universidades, en modalidad diurna, y que la malla curricular se encontrara disponible para su revisión. Durante el año 2012 se levantó una muestra correspondiente a $\mathrm{n}=212$ mallas, de un total de 601 programas de educación que se brindaban en ese momento. Para la segunda muestra, levantada durante el año 2018, el número de mallas de programas de pedagogía recopilados correspondió a $n=237$, de un total de 556 programas que cumplieron con los criterios mencionados. 


\subsection{Diseño de la investigación}

El presente trabajo corresponde a una investigación de tipo cuantitativo. Específicamente, un estudio documental con enfoque descriptivo longitudinal desarrollado en dos momentos:

- Durante el período de enero y julio de 2012.

- Durante el período de mayo y agosto de 2018, y revisada en noviembre de 2018.

Como procedimiento se analizaron las mallas curriculares de carreras de formación inicial de profesores de universidades e institutos profesionales de Chile. La elección de estos dos momentos se relaciona con:

- Publicación de estándares orientadores para las carreras de pedagogía en el año 2012, los que debieron influir en las características de la formación de profesores.

- Procesos de rediseño o renovación curricular, en el contexto de Proyectos de Mejoramiento Institucional o Convenios Marco.

- Un período de implementación de un Plan de Estudios renovado, considerando los cinco años para realizar la acreditación de la carrera.

\subsection{Instrumento}

Para el análisis documental se empleó una planilla de cálculo en la cual se almacenó la información levantada. Este archivo consolidado permitió la creación de una base de datos, la cual comprende nueve campos, presentados en la Tabla 2:

Tabla 2

Estructura de base de datos

\begin{tabular}{|c|c|c|}
\hline$N$ & Campos & Descripción del campo \\
\hline 1 & Nombre de la institución & $\begin{array}{l}\text { Contiene el nombre de la institución de } \\
\text { formación académica a la cual pertenece la } \\
\text { carrera revisada. }\end{array}$ \\
\hline 2 & Nombre de la carrera & $\begin{array}{l}\text { Se transcribe el nombre exacto de la } \\
\text { carrera analizada. }\end{array}$ \\
\hline 3 & Nombre de la disciplina principal & $\begin{array}{l}\text { A partir del nombre de la carrera, se } \\
\text { identifica la o las disciplinas que aborda el } \\
\text { programa. }\end{array}$ \\
\hline 4 & $\begin{array}{l}\text { Nombre de la disciplina secundaria de la } \\
\text { carrera }\end{array}$ & $\begin{array}{l}\text { A partir del nombre de la carrera, se } \\
\text { identifica una segunda disciplina que } \\
\text { aborda el programa. }\end{array}$ \\
\hline 5 & $\begin{array}{l}\text { Enlace a la página web o documento con la } \\
\text { malla de la carrera }\end{array}$ & $\begin{array}{l}\text { Se añade la dirección URL en la cual se } \\
\text { puede acceder a la malla del programa. }\end{array}$ \\
\hline 6 & Número de semestres de la carrera & $\begin{array}{l}\text { Se especifica el número de semestres que } \\
\text { considera la carrera. }\end{array}$ \\
\hline
\end{tabular}




\begin{tabular}{lll}
\hline $7 \quad$ Número de las asignaturas TIC & $\begin{array}{l}\text { Se informa sobre el número de asignaturas } \\
\text { que hacen referencia específica a las TIC y }\end{array}$ \\
& que forman parte de las asignaturas \\
& obligatorias de la carrera. No se \\
& consideran, por tanto, las asignaturas \\
& optativas del programa. \\
\hline $8 \quad$ Nombre de la asignatura TIC & Se transcribe el nombre de la asignatura \\
& TIC identificada. \\
\hline $9 \quad$ Semestre de la asignatura TIC de la carrera & $\begin{array}{l}\text { Se añade el número del semestre en que se } \\
\text { encuentra la asignatura. }\end{array}$ \\
\hline
\end{tabular}

Fuente: Elaboración propia.

En el caso de que el programa formativo incluya más de una asignatura TIC, se incorporan más columnas con las características de las columnas 8 y 9.

\subsection{Procedimientos}

\subsubsection{Periodo enero - julio 2012}

Elaboramos un listado de carreras de pedagogía y seleccionamos aquellas que forman profesores de educación media. A partir de este listado, procedimos a buscar en los sitios web de cada institución la malla curricular. Del total de 250 carreras de pedagogía que cumplían con los tres primeros criterios mencionados, se eliminaron los programas no concurrentes y se descartaron aquellos a los que no se pudo acceder a la malla, recopilando finalmente un total de 212 programas formativos.

Haciendo uso de la información de las mallas curriculares y utilizando una hoja de cálculo, registramos los datos siguiendo el modelo de base de datos. En este paso solo consideramos aquellas asignaturas TIC que están explícitamente mencionadas en el plan de formación y que aportan al perfil de egreso de la carrera, incluyendo las asignaturas de carácter transversal para todas las carreras de la institución formadora. Por tanto, aquellas asignaturas optativas o electivas no se consideraron para ser incorporadas en la base de datos.

Una vez que se dispuso del total de los programas, se clasificaron por disciplina formativa en los siguientes grupos, tal como se muestra en la Tabla 3:

Tabla 3

Clasificación de las asignaturas

\begin{tabular}{llll}
\hline $\mathbf{N}$ & Nombre grupo & Asignaturas \\
\hline 1 & Cs. Naturales & Incluye Pedagogía en Biología, Pedagogía en Física, \\
& Pedagogía en Química y afines.
\end{tabular}




\begin{tabular}{|c|c|c|}
\hline 2 & Cs. Sociales & $\begin{array}{l}\text { Incluye Pedagogía en Historia, Pedagogía en Geografía, } \\
\text { Pedagogía en Ciencias Sociales y afines. }\end{array}$ \\
\hline 3 & Lenguaje & $\begin{array}{l}\text { Incluye Pedagogía en Lengua Castellana, Pedagogía en } \\
\text { Lenguaje y Comunicación y afines. }\end{array}$ \\
\hline 4 & Matemáticas & $\begin{array}{l}\text { Incluye Pedagogía en Matemáticas, Pedagogía en } \\
\text { Matemática y Computación, Pedagogía en Matemática y } \\
\text { Física y afines. }\end{array}$ \\
\hline 5 & Idioma extranjero & Incluye Pedagogía en Inglés. \\
\hline 6 & Educación Física & $\begin{array}{l}\text { Incluye Pedagogía en Educación Física, Pedagogía en } \\
\text { Educación Física, Deportes y Recreación y afines. }\end{array}$ \\
\hline 7 & Artes & $\begin{array}{l}\text { Incluye Pedagogía en Artes Visuales, Pedagogía en Música, } \\
\text { Pedagogía en Artes Plásticas y afines. }\end{array}$ \\
\hline 8 & Religión y Filosofía & $\begin{array}{l}\text { Incluye Pedagogía en Religión, Pedagogía en Filosofía y } \\
\text { afines. }\end{array}$ \\
\hline
\end{tabular}

Fuente: Elaboración propia.

Para el levantamiento de información de la segunda muestra, se trabajó entre los meses de mayo y agosto empleando Google Drive Spreadsheet, a fin de realizar el proceso de forma colaborativa por parte del equipo investigador. Posteriormente, se descargó el archivo para analizar los datos empleando Microsoft Excel.

El proceso de levantamiento de información se realizó de forma similar al período anterior, manteniendo los criterios de selectividad. Esto incluyó, por tanto, acceder a los sitios web oficiales de las instituciones y, a partir de ello, a las páginas web o documentos PDF que contienen la información oficial y actualizada de cada carrera de pedagogía. En el caso de programas formativos que se realizan en múltiples sedes, pero con un mismo programa, solo se consideró una versión del mismo.

Una vez levantada la información, se realizó una segunda revisión aleatoria durante el mes de agosto a un $10 \%$ del total de los registros, a fin de evaluar la calidad de los datos obtenidos. Esta acción permitió modificar algunos enlaces incorrectos. Durante el mes de noviembre de 2018 se actualizó la base de datos considerando los cambios en cada carrera. Finalmente, y luego de disponer del total de los programas, se crearon los grupos de forma similar a la muestra del año 2012. 


\section{Resultados}

Tras realizar el registro de los datos, se clasificaron los programas de acuerdo a las asignaturas del currículo escolar y disciplinas de apoyo, generando ocho grupos de programas de formación. Se distribuyeron las carreras en los grupos creados, tal como muestra la Tabla 4. En el caso de algunos programas, a causa de su doble salida, se pueden situar en dos grupos distintos. Lo anterior implica que la suma del número de programas por grupo es superior al total de programas considerados para el estudio.

Tabla 4

Número de programas de formación analizados por grupo disciplinar en años 2012 y 2018

\begin{tabular}{lcc}
\hline Grupo disciplinar & Programas año 2012 & Programas año 2018 \\
\hline Ciencias Naturales & 27 & 40 \\
\hline Ciencias Sociales & 30 & 25 \\
\hline Lenguaje & 25 & 24 \\
\hline Matemáticas & 25 & 33 \\
\hline Idioma extranjero & 37 & 55 \\
\hline Educación Física & 28 & 36 \\
\hline Artes & 19 & 20 \\
\hline Religión y Filosofía & 21 & 16
\end{tabular}

Fuente: Elaboración propia.

Los datos fueron analizados en dos niveles: a nivel general, considerando la totalidad de los programas, y a nivel específico, para cada uno de los grupos establecidos. El análisis consistió en la comparación de:

a) El número de asignaturas TIC para el total de carreras analizadas y para cada grupo disciplinar, entre los años 2012 y 2018.

b) El promedio de asignaturas TIC para el total de programas y por grupo disciplinar, entre los años 2012 y 2018.

A continuación, se presentan y discuten los resultados tanto a nivel general como por grupo disciplinar de los datos anteriormente mencionados.

\subsection{Resultados generales}

La Tabla 5 presenta los resultados estadísticos generales de la investigación, incluyendo el número total de carreras y de carreras con al menos 1 asignatura TIC, el porcentaje de programas con al menos 1 asignatura TIC, el promedio de asignaturas TIC para el total de programas y el promedio de asignaturas TIC para los programas con al menos 1 asignatura TIC. Se incluye el porcentaje de variación para los tres últimos datos. 
Tabla 5

Datos estadísticos descriptivos

\begin{tabular}{lccc}
\hline Ítem & Año 2012 & Año 2018 & Variación \\
\hline Número total de carreras & 212 & 237 & \\
\hline Carreras con al menos 1 asignatura TIC & 133 & 167 & \\
\hline $\begin{array}{l}\text { Porcentaje de programas con al menos 1 } \\
\text { asignatura TIC }\end{array}$ & $62,7 \%$ & $70,5 \%$ & $+12,4 \%$ \\
\hline $\begin{array}{l}\text { Promedio de asignaturas TIC para el total de } \\
\text { programas }\end{array}$ & 0,88 & 1,14 & $+29,5 \%$ \\
\hline $\begin{array}{l}\text { Promedio de asignaturas TIC para los programas } \\
\text { con al menos 1 asignatura TIC }\end{array}$ & 1,39 & 1,60 & $+15,1 \%$ \\
\hline
\end{tabular}

Fuente: Elaboración propia.

Al analizar la presencia de asignaturas TIC en los planes de formación, podemos identificar una variación disímil. En la Tabla 6 se presenta el número y porcentaje de asignaturas TIC para cada año, junto a la variación porcentual:

Tabla 6

Número de programas y porcentaje del total de programas con asignaturas TIC

\begin{tabular}{lccccc}
\hline Criterio & N 2012 & \% 2012 & N 2018 & \% 2018 & Variación porcentual \\
\hline Con 1 asignatura TIC & 99 & $74,44 \%$ & 98 & $58,68 \%$ & $-21,2 \%$ \\
\hline Con 2 asignaturas TIC & 25 & $18,8 \%$ & 52 & $31,14 \%$ & $+65,6 \%$ \\
\hline Con 3 asignaturas TIC & 3 & $2,26 \%$ & 8 & $4,79 \%$ & $+111,9 \%$ \\
\hline Con 4 asignaturas TIC & 3 & $2,26 \%$ & 3 & $1,8 \%$ & $-20,4 \%$ \\
\hline Con 5 asignaturas TIC & 2 & $1,5 \%$ & 5 & $2,99 \%$ & $+99 \%$ \\
\hline Con 6 asignaturas TIC & 1 & $0,75 \%$ & 1 & $0,6 \%$ & $-20 \%$ \\
\hline
\end{tabular}

Fuente: Elaboración propia.

\subsection{Resultados por grupo disciplinar}

El número de asignaturas para cada grupo es diverso, no obstante, es posible realizar algunas comparaciones. Los resultados se presentan realizando la comparación entre idénticos grupos disciplinares para ambos períodos, y entre los diferentes grupos formativos dentro de un mismo período.

Como se observa en la Tabla 7, el número total de programas por grupo disciplinar considerado en la muestra ha variado, especialmente en Ciencias Naturales e Idioma extranjero. 
ANÁLISIS COMPARATIVO DE LAS ASIGNATURAS TIC EN LA FORMACIÓN INICIAL DE PROFESORES EN CHILE ENTRE 2012 Y 2018

Tabla 7

Número de asignaturas TIC por grupo disciplinar, años 2012 y 2018

\begin{tabular}{|c|c|c|c|c|c|c|}
\hline \multirow[t]{2}{*}{ Grupo disciplinar } & \multicolumn{3}{|c|}{2012} & \multicolumn{3}{|c|}{2018} \\
\hline & $\begin{array}{l}\text { Total de } \\
\text { carreras }\end{array}$ & $\begin{array}{c}\text { Carreras con } \\
\text { asign. TIC }\end{array}$ & $\%$ & $\begin{array}{l}\text { Total de } \\
\text { carreras }\end{array}$ & $\begin{array}{c}\text { Carreras con } \\
\text { asign. TIC }\end{array}$ & $\%$ \\
\hline Ciencias Naturales & 27 & 16 & $59,3 \%$ & 40 & 29 & $72,5 \%$ \\
\hline Ciencias Sociales & 30 & 19 & $63,3 \%$ & 25 & 19 & $76 \%$ \\
\hline Lenguaje & 25 & 16 & $64 \%$ & 24 & 15 & $62,5 \%$ \\
\hline Matemáticas & 25 & 19 & $76 \%$ & 33 & 28 & $84,8 \%$ \\
\hline Idioma extranjero & 37 & 24 & $64,9 \%$ & 53 & 36 & $67,9 \%$ \\
\hline Educación Física & 28 & 19 & $67,9 \%$ & 36 & 25 & $69,4 \%$ \\
\hline Artes & 19 & 11 & $57,9 \%$ & 20 & 17 & $85 \%$ \\
\hline Religión y Filosofía & 21 & 9 & $42,9 \%$ & 16 & 7 & $43,8 \%$ \\
\hline
\end{tabular}

Fuente: Elaboración propia.

A partir de los datos presentados en la Tabla 7, podemos comparar ambos períodos. Para ello, calculamos el porcentaje de programas formativos que incluyen al menos una asignatura TIC, según grupo disciplinar. Los resultados se pueden ver en la Figura 1.

2012

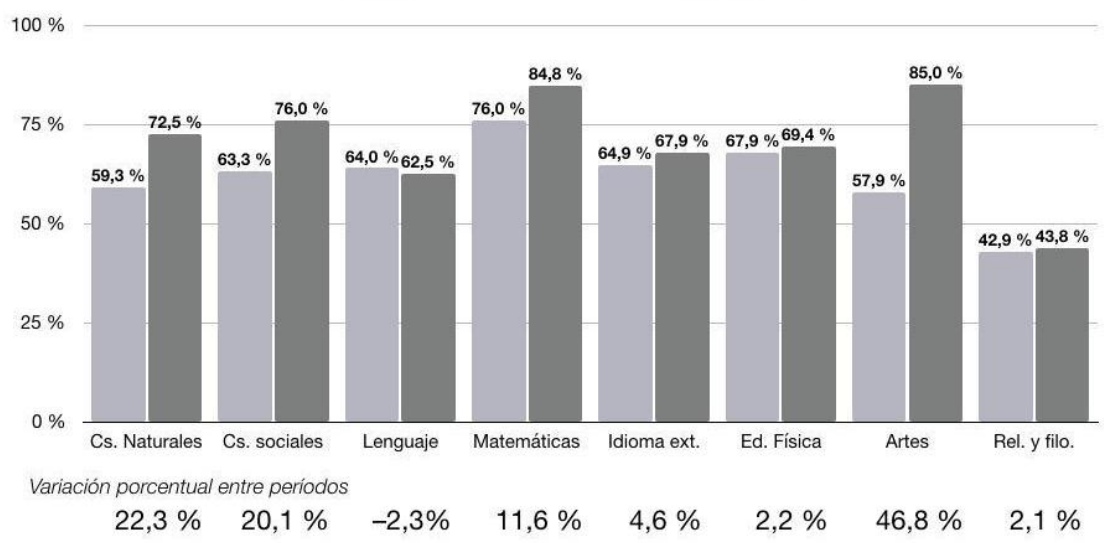

Figura 1. Comparativa entre 2012 y 2018 del porcentaje de programas con al menos 1 asignatura TIC según grupo disciplinar.

Fuente: Elaboración propia.

La Figura 1 nos muestra una variación positiva considerable de casi un 50\% en Artes. En el caso de Ciencias Naturales, Ciencias Sociales y Matemáticas, el aumento es menor, pero importante. En los grupos de Lenguaje, Idioma extranjero, Educación Física y Religión y Filosofía, la variación no es significativa, oscilando entre un $-2,2 \%$ y un $+4,6 \%$. 
Respecto a la comparativa entre 2012 y 2018 de los promedios de asignaturas TIC para el total de las carreras por grupo disciplinar, podemos ver los resultados en la Figura 2.

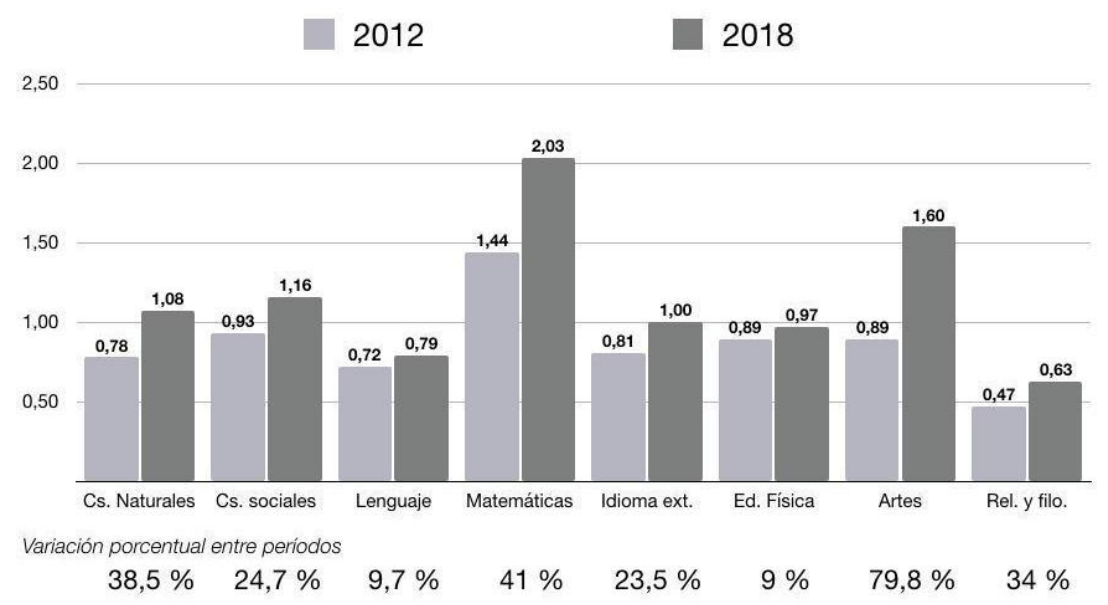

Figura 2. Comparativa entre 2012 y 2018 del promedio de asignaturas TIC para el total de programas de un grupo disciplinar.

Fuente: Elaboración propia.

Se observa que el grupo de Matemáticas presenta en ambos períodos el promedio más alto de asignaturas TIC. Por el contrario, el grupo de Religión y Filosofía presenta los promedios de asignatura TIC más bajos. Si comparamos ambos grupos, Matemáticas triplica el número de asignaturas frente el segundo.

También es destacable que en el período 2012, solo el grupo de Matemáticas supera el promedio de 1 asignatura. En el caso del período 2018, cinco grupos disciplinares superan el promedio de 1 asignatura.

Respecto a la variación interperíodos, los datos nos muestran que ella es positiva en el promedio de asignaturas TIC en todos los grupos. La variación es especialmente importante en el grupo de Artes, con casi un $80 \%$ de aumento, seguido por Matemáticas, Ciencias Naturales y Religión y Filosofía. Por el contrario, los grupos de Lenguaje y Educación Física no presentan una gran variación.

En el caso de los valores para las carreras que tienen al menos 1 asignatura TIC, observamos los resultados que nos presenta la Figura 3. 


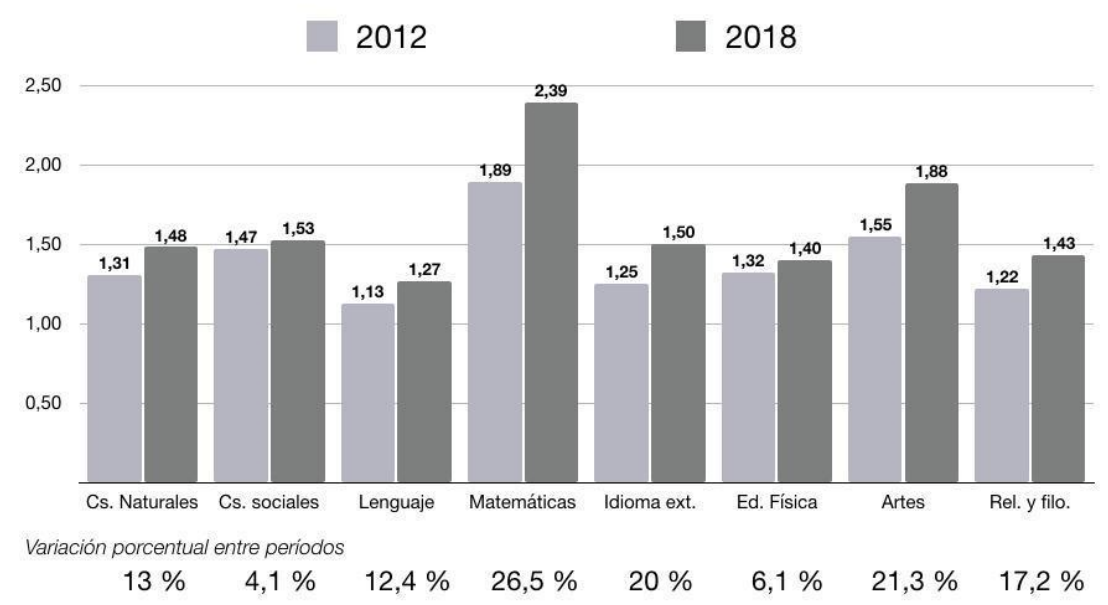

Figura 3. Comparativa entre 2012 y 2018 del promedio de asignaturas TIC para los programas con al menos 1 asignatura TIC según grupo disciplinar.

Fuente: Elaboración propia.

En el período 2012, los valores se encuentran en un rango entre 1,13 y 1,89 asignaturas, destacando el grupo de Matemáticas, seguido por el grupo de Artes. Lenguaje y Religión y Filosofía son los grupos formativos que en este período tiene el menor promedio de asignaturas TIC para los programas que incluyen asignaturas TIC.

Para el período 2018, las medias se encuentran en un rango entre 1,27 y 2,39 asignaturas. Tal como en el período anterior, destaca el grupo de Matemáticas, seguido por el grupo de Artes. Los grupos con menor promedio son Lenguaje y Educación Física.

La variación de las medias para el caso de las asignaturas que disponen de una o más asignaturas TIC, es menor que para la totalidad de carreras de cada grupo disciplinar. El mayor aumento lo experimentan los grupos de Matemáticas y de Artes. El grupo de Ciencias Sociales, siendo el de menor crecimiento, lo hace en un $4,1 \%$. 


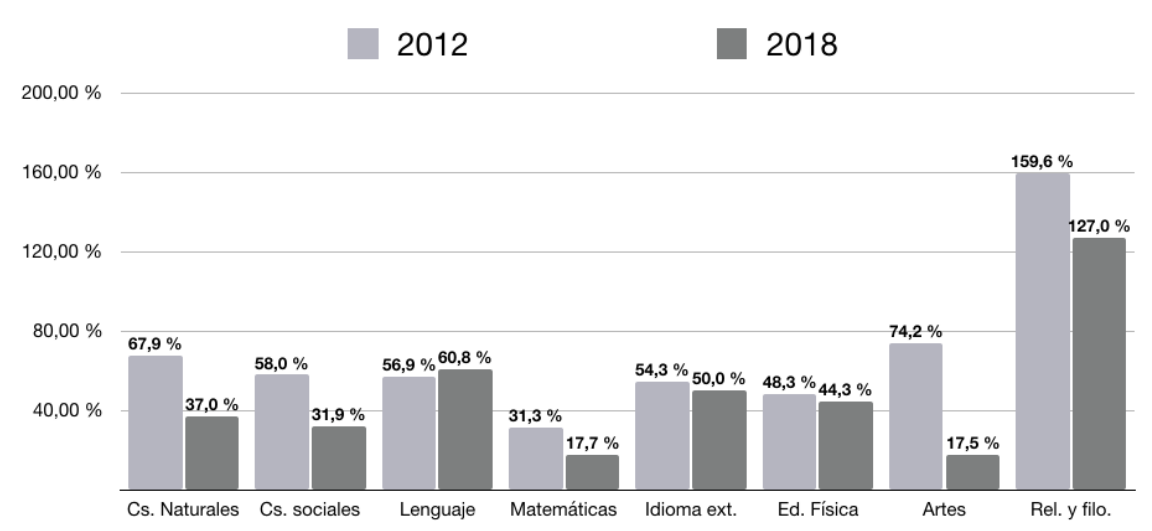

Figura 4. Comparativa entre 2012 y 2018 de la diferencia porcentual entre promedios de asignaturas TIC para el total y para los programas con al menos 1 asignatura TIC según grupo disciplinar.

Fuente: Elaboración propia.

Los datos de la Figura 4 muestran una disminución generalizada, aunque con valores porcentuales diferentes, de la diferencia entre los promedios de los programas que incluyen el uso de las TIC y el promedio del total.

\section{Discusión y conclusiones}

Los resultados generales nos muestran un incremento, entre el 2012 y 2018, en el porcentaje de programas de formación de profesores que cuentan con, al menos, 1 asignatura TIC. Este dato de crecimiento, un 12,4\%, estaría vinculado a un aumento en la preocupación por la presencia de las TIC: aumentó de 6 de cada 10, a 7 de cada 10 programas de formación inicial de profesores que incluye al menos una asignatura TIC en su currículo. Estos datos coinciden con el escenario presentado por Brun e Hinostroza (2011) y Garrido et al. (2008), a nivel nacional, y por Tondeur et al. (2016) y Herrada Valverde y Herrada Valverde (2011), a nivel internacional.

Es relevante que los promedios de asignaturas TIC reflejan un aumento porcentual de casi un $30 \%$ para el total de carreras, y de un 15\% para aquellas carreras que cuentan con al menos 1 asignatura TIC. Esto sería un indicador tanto de un aumento en el número de asignaturas TIC a partir de los rediseños o renovaciones de los programas de formación inicial de profesores, como de una incorporación mayor de las TIC en los programas de formación de creación reciente. Esto es coincidente con los procesos de reforma en la formación de profesores que mencionan Rizza (2011) y Herrada Valverde y Herrada Valverde (2011). 
Reflejo de esta variación positiva en el período 2012-2018 es tanto el número como el porcentaje de programas con 2 y 3 asignaturas TIC. También lo es la disminución de los programas con 1 asignatura TIC. Estos datos podrían ser consistentes con los procesos de renovación curricular, y en especial con la incorporación de los Estándares Orientadores para la Formación Inicial de Profesores (MINEDUC, 2012a, 2012b, 2012c) que se han desarrollado en los últimos años.

Respecto a la formación en TIC de futuros profesores, es posible que, mientras no se encuentre certificada ni posea implicancias para el ejercicio de la profesión, se mantenga su carácter optativo o complementario para la formación inicial. Se requeriría, por tanto, implementar políticas formativas reguladoras que impliquen exigencias de certificación a los centros formativos o para el profesorado que egresa. Este enfoque propiciaría un aumento en la presencia de las TIC en la FIP en la medida que las instituciones se vieran obligadas a ejecutar tareas para asegurar el cumplimiento de estas regulaciones (Goldstein \& Tesler, 2017).

Destacamos que, en ambos períodos, un 90\% de las carreras con asignaturas TIC dispone de 1 o 2 de ellas. No obstante su relevancia, es necesario preguntarse, como Flores-Lueg y Roig Vila (2016), si tal número de asignaturas representa una cifra adecuada de asignaturas TIC para un programa simultáneo, de forma que permita alcanzar los conocimientos y habilidades necesarios para su transferencia a su propio aprendizaje y a su labor en el contexto educativo.

En el caso de la variación entre los grupos disciplinares, los resultados son dispares y reflejarían una toma de decisiones importante. Destaca el aumento de un $46,8 \%$ en el caso del grupo de Artes, seguido por Cs. Naturales y Cs. Sociales. Este aumento podría reflejar un importante cambio en la concepción de estos programas formativos y su relación con la tecnología (Parcerisa, 2010), aspecto que podría beneficiarse de los procesos de renovación curricular. No obstante, la variación positiva del porcentaje en algunos grupos disciplinares no reflejaría necesariamente un aumento en la presencia de las TIC, por cuanto habría que analizar la progresión específica de cada carrera.

En el caso del grupo disciplinar de Filosofía y Religión, este se mantiene con un bajo porcentaje de programas con asignaturas TIC. Matemáticas, por el contrario, se sitúa en ambos periodos con el porcentaje más alto de carreras con al menos una asignatura TIC. Si comparamos estos datos con los usos en la escuela (Labbé et al., 2012; Rončević Zubković, Pahljina-Reinić \& KolićVehovec, 2017), existiría correspondencia entre el nivel de integración TIC en la enseñanza escolar de las disciplinas y el número de asignaturas TIC durante la formación de profesores. 
Respecto al promedio de asignaturas TIC, se observa que en todos los grupos disciplinares esta cifra presenta un aumento. En el caso del promedio para las carreras con al menos 1 asignatura TIC, vemos que presenta un importante incremento, pero que es menor al crecimiento que se observa al comparar con los promedios para todos los programas. Esto podría deberse a un crecimiento de las asignaturas TIC a nivel interno más uniforme en los programas de pedagogía. Durante el período 2012, el rango en el que se encuentran los programas es menor que en el periodo $2018-\Delta$ de 0,76 versus $\Delta$ 1,12-, lo que podría implicar una brecha mayor de conocimientos TIC entre quienes egresan de una carrera que pertenece a un grupo disciplinar $\mathrm{u}$ otro.

Si se comparan las diferencias porcentuales entre los promedios de asignaturas TIC para el total de programas y para los programas que tienen al menos una asignatura TIC entre los dos períodos, se infiere que la brecha entre ambos promedios ha disminuido, en términos generales. No obstante ello, ha descendido de forma heterogénea, con grupos disciplinares que han pasado de un $74,2 \%$ de diferencia a un $17,5 \%$-tal es el caso de Artes- o con grupos como el de Matemáticas y Ciencias Sociales que han disminuido a valores cercanos a la mitad durante el período 2018.

En términos complementarios, sería importante investigar si las carreras que no cuentan con asignaturas TIC optan por un enfoque transversal para abordarlas en la formación. Se vincula con este aspecto aquellas asignaturas que son de carácter obligatorio para todas las carreras de una universidad y, por tanto, no poseen un enfoque hacia la práctica pedagógica.

Lo anterior es relevante pues, si consideramos lo que señalan Brun e Hinostroza (2011), es posible que muchos futuros profesores cuenten con escasas oportunidades de aprendizaje para desarrollar conocimientos y habilidades en el uso de las TIC. Coinciden con él Garrido et al. (2013), mencionando que los egresados de pedagogía experimentarían escasas oportunidades de aprendizaje innovador durante su FIP, con consecuencias en sus habilidades para desenvolverse en el contexto escolar. Esto es aún más interesante si se considera que los futuros profesores, a pesar de crecer en un contexto tecnológico, no necesariamente poseen habilidades especiales para desenvolverse en contextos digitales (Claro et al., 2018).

Por otra parte, los resultados son coherentes con lo señalado por De Aldama y Pozo (2016), Howard et al. (2015) y Labbé et al. (2012), en relación a que el uso de las TIC difiere en el profesor según su disciplina, dependiendo de cuestiones relacionadas con el área formativa, el perfil de egreso de cada carrera y las características de los programas de las asignaturas TIC. Estos 
elementos dan espacio para estudiar y profundizar en futuras investigaciones sobre el tema, con el fin de brindar una imagen más detallada de la integración de las TIC.

Finalmente, podemos señalar que la preocupación por la incorporación de asignaturas TIC en la formación de profesores es dispar, incluso en carreras de la misma especialidad ofrecida por distintas instituciones, coincidiendo con Silva (2017), en cuanto la incorporación de las TIC no ha permeado en el currículo de la formación docente de las instituciones de educación superior. Esperamos, por tanto, que la evaluación que realice una carrera de la forma en que se integran las TIC en su plan de estudios considere los datos presentados en esta investigación.

\subsection{Limitaciones de la investigación}

Los resultados y conclusiones de la presente investigación se encuentran limitados debido a los cambios en la oferta de carreras de pedagogía. Estos cambios están vinculados al cierre, renovación curricular y creación de nuevos programas, por lo que en algunos casos los datos considerados para la variación deberían suponerse como una descripción del momento.

\subsection{Proyecciones de la investigación}

El presente trabajo es parte de un proyecto que considera, en etapas siguientes, analizar y categorizar el contenido de los programas de asignaturas TIC de carreras de pedagogía, identificar las orientaciones de los formadores en TIC de futuros profesores y evaluar el impacto de la formación TIC en la FIP en las prácticas pedagógicas. 


\section{Referencias Bibliográficas}

Adnan, M., \& Tondeur, J. (2018, 25 al 29 de junio). Preparing the next generation for effective technology integration in education: Teacher educators' perspective. Actas de la Conferencia EdMedia + Innovate Learning. Conferencia llevada a cabo en Amsterdam, Países Bajos.

Arancibia Herrera, M. M., Casanova Seguel, R., \& Soto Caro, C. P. (2016). Concepciones de profesores sobre aprender y enseñar usando tecnologías. Ciencia, docencia y tecnología, 27(52). Recuperado desde http://www.pcient.uner.edu.ar/index.php/cdyt/article/download/162/198

Ascencio Ojeda, P., Garay Aguilar, M., \& Seguic Zeran, E. (2016). Formación Inicial Docente (FID) y Tecnologías de la Información y Comunicación (TIC) en la Universidad de Magallanes-Patagonia Chilena. Digital Education Review, (30), 123-134.

Ávalos, B. (2010). Formación Inicial Docente en Chile: calidad y políticas. En C. Bellei, D. Contreras \& P. Valenzuela (eds.). Ecos de la revolución pingüina. Avances, debates y silencios en la reforma educacional (pp. 257-284). Santiago: Universidad de Chile, UNICEF.

Barton, R., \& Haydn, T. (2006). Trainee teachers' views on what helps them to use information and communication technology effectively in their subject teaching. Journal of Computer Assisted Learning, 22(4), 257-272. Recuperado desde http://onlinelibrary.wiley.com/doi/10.1111/j.1365-2729.2006.00175.x/abstract

Brun, M., \& Hinostroza, J. E. (2011). Research on ICT integration for enhancing quality in teacher education: nationwide policy or global challenge? En E. Eisenschmidt \& E. Löfström (eds.). Developing quality cultures in teacher education: expanding horizons in relation to quality assurance (pp. 99-118). Tallin: Vali Press oü. https://dspace.ut.ee/bitstream/handle/10062/40578/Archimedes_teacher_educati on. pdf?sequence=1\&isAllowed=y

Chousa Cortés, C., Martínez-Figueira, M. E., \& Raposo-Rivas, M. (2017). Las TIC para la intervención educativa en TDAH: un estudio bibliométrico. Perspectiva educacional, 
56(3) 142-161. Recuperado desde http://perspectivaeducacional.cl/index.php/peducacional/article/view/521/2533

Claro, M., Salinas, A., Cabello-Hutt, T., San Martín, E., Preiss, D. D., Valenzuela, S., \& Jara, I. (2018). Teaching in a Digital Environment (TIDE): Defining and measuring teachers' capacity to develop students' digital information and communication skills. Computers \& Education, 121, 162-174. doi:10.1016/j.compedu.2018.03.001

Comisión Nacional de Acreditación. (2007). Criterios de evaluación de carreras de educación. Chile. Recuperado desde https://www.cnachile.cl/Criterios\%20de\%20carreras/CriteriosEducacionFINAL1.pdf

Comisión Nacional de Acreditación. (2017). Criterios específicos de evaluación para la acreditación de programas regulares de pedagogía. Chile. Recuperado desde https://www.cnachile.cl/noticias/SiteAssets/paginas/PEDAGOGÍAS-NUEVODESAFÍO/Documento\%20de\%20trabajo\%20Criterios\%20Programas\%20Regulares\%2 OPedagog\%C3\%ADa.pdf

Comisión Nacional de Acreditación. (2018). Carreras de pedagogía: análisis de fortalezas y debilidades en el escenario actual. Chile. Recuperado desde https://investigacion.cnachile.cl/archivos/cna/documentos/Carreras-depedagogia_Serie-Estudios-CNA.pdf

De Aldama, C., \& Pozo, J. I. (2016). How are ICT used in the classroom? A study of teachers' beliefs and uses. Electronic Journal of Research in Educational Psychology, 14(2), 253-286. doi:10.14204/ejrep.39.15062

ENLACES. (2008). Estándares TIC para la formación inicial docente: una propuesta en el contexto chileno. Santiago de Chile: Autor.

Enochsson, A.-B., \& Rizza, C. (2009). ICT in Initial Teacher Training: Research Review. Recuperado desde http://www.oecd-ilibrary.org/education/ict-in-initial-teacher-trainingresearch-review_220502872611

Flores-Lueg, C., \& Roig Vila, R. (2016). Percepción de estudiantes de Pedagogía sobre el desarrollo de su competencia digital a lo largo de su proceso formativo. Estudios 
pedagógicos(Valdivia), $\quad$ XLII(3), 129-148. Recuperado desde https://scielo.conicyt.cl/pdf/estped/v42n3/art07.pdf

Forkosh-Baruch, A. (2018). Preparing Preservice Teachers to Transform Education with Information and Communication Technologies. En J. Voogt, G. A. Knezek, R. Christensen, \& K.-W. Lai (Eds.). Second Handbook of Information Technology in Primary and Secondary Education (pp. 415-432). Switzerland: Springer.

García-Valcárcel, A. \& Martín del Pozo, M. (2016). Análisis de las competencias digitales de los graduados en titulaciones de maestro. RELATEC: Revista Latinoamericana de Tecnología Educativa, 15(2), 155-168. doi:10.17398/1695-288X.15.2.155

Garrido, J. M., Gros, B., Rodríguez, J., Silva, J., \& Nervi, H. (2008). Más allá de laptops y pizarras digitales: la experiencia chilena de incorporación de TIC en la formación inicial de docentes. Calidad en la Educación, 29, 195-209. http://dx.doi.org/10.31619/caledu.n29.193

Garrido, J., Contreras, D., \& Miranda, C. (2013). Análisis de la disposición pedagógica de los futuros profesores para usar las TIC. Estudios pedagógicos (Valdivia), 39(ESPECIAL), 59-74. Recuperado desde https://scielo.conicyt.cl/scielo.php?pid=S071807052013000300005\&script=sci_arttext

Gibson, S., Moline, T., \& Dyck, B. (2011). What Are Education Students' Perceptions of the Role of Technology in Social Studies Pedagogy? Alberta Journal of Educational Research, 57(1), 73-87.

Gobierno de Chile. (2015). Agenda Digital 2020. Chile. Recuperado desde http://www.agendadigital.gob.cl/files/Agenda\%20Digital\%20Gobierno\%20de\%20Chi le\%20-\%20Noviembre\%202015.pdf

Goldstein, O., \& Tesler, B. (2017). The Impact of the National Program to Integrate ICT in Teaching in Pre-Service Teacher Training. Interdisciplinary Journal of e-Skills and Lifelong Learning, 13, 151-166. doi:10.28945/3876 
Herrada Valverde, R. I., \& Herrada Valverde, G. (2011). Adaptación de los estudios de magisterio al EEES: Las TIC en los nuevos planes de estudio. EDUTEC. Revista Electrónica de Tecnología Educativa, (36), 1-12. doi:10.21556/edutec.2011.36.405

Howard, S. K., Chan, A., \& Caputi, P. (2015). More than beliefs: Subject areas and teachers' integration of laptops in secondary teaching. British Journal of Educational Technology, 46(2), 360-369 doi:10.1111/bjet.12139

Instefjord, E., \& Munthe, E. (2016). Preparing pre-service teachers to integrate technology: an analysis of the emphasis on digital competence in teacher education curricula. European Journal of Teacher Education, 39(1), 77-93. doi:10.1080/02619768.2015.1100602

Jara, I. (2007). Los Desafíos de las Políticas de TIC para Escuelas. Revista Pensamiento Educativo, 40(1), 373-390.

Jara, I., \& Hinostroza, J. E. (2014). Análisis de Política TIC para el Sistema Escolar en Chile: Situación actual y perspectivas. Informe final. Temuco: Universidad de la Frontera. Recuperado desde

https://biblioteca.digital.gob.cl/bitstream/handle/123456789/334/AnalisisdePolitica TICparaelSistemaEscolarenChile_2014.pdf?sequence=1\&isAllowed=y

Kontkanen, S. (2018). Starting points of Pre-service teachers' technological pedagogical content knowledge (TPACK) - Introducing a Proto-TPACK model (126). Joensuu: University of Eastern Finland.

Labbé, C., Matamala, C., Astudillo, G., \& Hinostroza, J. E. (2012). Uso de TIC por parte de profesores de Lenguaje, Matemática y Ciencias en educación media: Un estudio exploratorio. Actas del Segundo Congreso Interdisciplinario de Investigación en Educación. Congreso llevado a cabo en Santiago de Chile.

Marcelo, C. (2016). Estado del arte internacional de los modelos de formación inicial docente: FID. Sevilla: Universidad de Sevilla. Recuperado desde http://www.upla.cl/innovacioncurricular/wp-content/uploads/2012/06/InformeFinal-AT-Internacional.pdf 
Martínez, R. E., Leite, C., \& Reis, A. M. (2016). Políticas de integración de tecnologías y Formación Inicial de Maestros en Uruguay. Archivos analíticos de políticas educativas, 24(22), 127. doi:10.14507/epaa.24.2017

Ministerio de Educación. (2012a). Estándares orientadores para carreras de pedagogía en Educación Básica (Standard Definition ed.). Santiago, Chile: Autor. Recuperado desde http://www.cpeip.cl/wp-content/uploads/2016/07/librobasicaokdos.pdf

Ministerio de Educación. (2012b). Estándares orientadores para carreras de pedagogía en Educación Media (Standard Definition ed.). Santiago, Chile: Autor. Recuperado desde http://www.cpeip.cl/usuarios/cpeip/File/librosestandaresvale/libromediafinal.pdf

Ministerio de Educación. (2012c). Estándares orientadores para carreras de pedagogía en Educación Parvularia. Santiago, Chile: Autor.

Mouza, C., Karchmer-Klein, R., Nandakumar, R., Yilmaz Ozden, S., \& Hu, L. (2014). Investigating the impact of an integrated approach to the development of preservice teachers' technological pedagogical content knowledge (TPACK). Computers \& Education, 71, 206-221. doi:10.1016/j.compedu.2013.09.020

Muñoz, R. E., \& Ortega, J. A. (2015). ¿Tienen la banda ancha y las TIC un impacto positivo sobre el rendimiento escolar? Evidencia para Chile. El trimestre económico, 82(325), 53-87.

Parcerisa, A. (2010). Los materiales didácticos como recurso en la acción comunitaria. En M. Area Moreira, A. Parcerisa, \& J. Rodríguez (Eds.). Materiales y recursos didácticos en contextos comunitarios (Vol. 1, pp. 15-30). España: GRAÓ.

Rebolledo Gómez, T. (2015). La formación inicial del profesorado de educación primaria y secundaria en Alemania, España, Finlandia y Reino Unido. Estudio comparado. Revista Española de Educación Comparada, 25, 129-148. doi:10.5944/reec.25.2015.14787

Rizza, C. (2011). ICT and Initial Teacher Education: National Policies. OECD Education Working Papers, 61. doi:10.1787/5kg57kjj5hs8-en

Røkenes, F. M., \& Krumsvik, R. J. (2016). Prepared to teach ESL with ICT? A study of digital competence in Norwegian teacher education. Computers \& Education, 97, 1-20. doi:10.1016/j.compedu.2016.02.014 
ANÁLISIS COMPARATIVO DE LAS ASIGNATURAS TIC EN LA FORMACIÓN INICIAL DE PROFESORES EN CHILE ENTRE 2012 Y 2018

Rončević Zubković, B., Pahljina-Reinić, R., \& Kolić-Vehovec, S. (2017). Predictors of ICT Use in Teaching in Different Educational Domains. European Journal of Social Sciences Education and Research, 11(2), 145-154. doi:10.26417/ejser.v11i2.p145-154

Silva, J. (2017). Inserción de TIC en pedagogías del área de las humanidades en una universidad chilena. Psicología, Conocimiento y Sociedad, 7(2), 80-96. doi:10.26864/pcs.v7.n2.6

Silva, J., Gros, B., Garrido, J. M., \& Rodríguez, J. (2006). Estándares en tecnologías de la información y la comunicación para la formación inicial docente: situación actual y el caso chileno. Revista Iberoamericana de Educación, 38(3), 1-16. doi:10.35362/rie3832658

Tondeur, J., Van Braak, J., Siddiq, F., \& Scherer, R. (2016). Time for a new approach to prepare future teachers for educational technology use: Its meaning and measurement. Computers \& Education, 94, 134-150. doi:10.1016/j.compedu.2015.11.009

Webb, M., \& Cox, M. (2004). A review of pedagogy related to information and communications technology. Technology, Pedagogy and Education, 13(3), 235-286. doi:10.1080/14759390400200183 